\title{
Effect of intensive counselling on the quality of dietary fats in pregnant women at high risk of gestational diabetes mellitus
}

\author{
Eeva Korpi-Hyövälti ${ }^{1 *}$, Ursula Schwab ${ }^{2,3}$, David E. Laaksonen ${ }^{2,4}$, Hilpi Linjama ${ }^{5}$, Seppo Heinonen ${ }^{6}$ \\ and Leo Niskanen ${ }^{7,8}$ \\ ${ }^{1}$ Department of Internal Medicine, Seinäjoki Central Hospital, 60220 Seinäjoki, Finland \\ ${ }^{2}$ Institute of Clinical Medicine, Internal Medicine, Kuopio University Hospital, 70211 Kuopio, Finland \\ ${ }^{3}$ Department of Clinical Nutrition, Institute of Public Health and Clinical Nutrition, University of Eastern Finland, \\ Kuopio Campus, 70211 Kuopio, Finland \\ ${ }^{4}$ Institute of Biomedicine, Physiology, University of Eastern Finland, Kuopio Campus, 70211 Kuopio, Finland \\ ${ }^{5}$ Primary Health Care and Health Promotion Unit, Seinäjoki Central Hospital, 60220 Seinäjoki, Finland \\ ${ }^{6}$ Department of Obstetrics and Gynecology, Kuopio University Hospital, 70211 Kuopio, Finland \\ ${ }^{7}$ Central Hospital of Central Finland, 40620 Jyväskylä, Finland \\ ${ }^{8}$ School of Medicine, University of Eastern Finland, Kuopio Campus, 70211 Kuopio, Finland
}

(Submitted 12 April 2011 - Final revision received 24 August 2011 - Accepted 12 October 2011 - First published online 18 November 2011)

\begin{abstract}
As part of a feasibility study to prevent gestational diabetes mellitus (GDM), we evaluated the effect of an intensive dietary therapy on quality of diet, weight gain and birth weight in women at high risk of GDM. Women with risk factors for GDM ( $n$ 54) were randomly assigned from April 2005 to May 2006 to a lifestyle intervention group ( $n$ 27) including dietary advice six times during pregnancy or to a close follow-up group $(n$ 27) in a community-based setting in Finland. Dietary intake was recorded three times during pregnancy using $4 \mathrm{~d}$ food records. The main outcome was the incidence of GDM. The secondary outcomes were the changes in nutrient intake, weight gain and birth weight. Overall, seventeen (65\%) women in the intervention group and eighteen (69\%) women in the close follow-up group returned all three food records. PUFA intake increased $(P=0 \cdot 008)$ during pregnancy in the intervention as compared to the close follow-up group. There were no clear differences in the changes of saturated fat or fibre intake between the groups. Intensive dietary education resulted in a somewhat lower weight gain during pregnancy $(P=0.062)$ and higher birth weights of the infants $(P=0 \cdot 047)$ without an effect on macrosomia as compared to the close follow-up group. Individualised counselling by a clinical nutritionist as part of a lifestyle intervention improved the quality of dietary fat intake in pregnant women at high risk of GDM.
\end{abstract}

Key words: Lifestyle intervention: Gestational diabetes mellitus: Nutrient intake: Fat quality

Type 2 diabetes can be prevented or delayed by overall lifestyle changes including increased physical activity, decreased dietary intake of total and saturated fat, and modest weight loss in high-risk individuals, based on findings in randomised, controlled studies ${ }^{(1,2)}$. There is still controversy over the optimal balance between carbohydrate and fat intake and the role of the quality of dietary fat in the prevention or treatment of gestational diabetes mellitus (GDM).

Most previous studies have been non-randomised comparisons of the intake of dietary fat and carbohydrate among pregnant women with normal glucose tolerance, impaired glucose tolerance and GDM. Moses et $a l^{(3)}$ found in an observational cohort study that a diet high in fats may increase the risk for recurrence of GDM. Increased polyunsaturated fat intake was associated with a reduced incidence of glucose intolerance during pregnancy, in an observational study of Chinese women $^{(4)}$. In another observational study, saturated fat intake was independently associated with the development of gestational glucose abnormalities, especially so in the absence of conventional risk factors ${ }^{(5)}$. Saldana et al. ${ }^{(6)}$ found an association between increased total fat intake and the development of glucose abnormalities in pregnancy, in an observational study.

As part of a feasibility study, we carried out a randomised, controlled trial comparing a lifestyle intervention group with a close follow-up group of women at high risk of GDM since early pregnancy, as described elsewhere ${ }^{(7)}$. We evaluated the effect of an intensive dietary therapy on

Abbreviations: E\%, percentage of energy; GDM, gestational diabetes mellitus.

*Corresponding author: Dr E. Korpi-Hyövälti, fax +358 6415 4291, email eeva.korpi-hyovalti@epshp.fi 
quality of diet, weight gain and birth weight in women at a high risk of GDM.

\section{Research design and methods}

We evaluated the effect of dietary counselling by a clinical nutritionist on intake of energy, fat quantity and quality, intake of fibre and other macro- and micronutrients compared with the close follow-up group with conventional dietary advice based on national recommendations ${ }^{(8)}$, in a single session given by out-patient maternity clinics. This was an open, randomised and controlled study with two participating Finnish rural municipalities in South Ostrobothnia: Kauhajoki and Lapua. The protocol was approved by the ethics committee of South Ostrobothnia Hospital District in Seinäjoki, Finland. It was in accordance with the Helsinki declaration. All women participating in the trial gave written informed consent.

\section{Subjects}

A $2 \mathrm{~h}$ oral glucose tolerance test was offered to all women in the first contact with maternal healthcare units during gestational weeks 8-12. If the women had one or more risk factors (BMI $>25 \mathrm{~kg} / \mathrm{m}^{2}$, previous history of GDM or birth of child $>4.5 \mathrm{~kg}$, age $>40$ years, family history of diabetes, i.e. parents, children, siblings or grandparents) or if the venous plasma glucose concentration after $12 \mathrm{~h}$ overnight fasting was $4.8-5.5 \mathrm{mmol} / 1$ and the $2 \mathrm{~h}$ oral glucose tolerance test plasma glucose $<7.8 \mathrm{mmol} / 1$, they were recruited to the intervention.

Of the initial group of subjects ( $n$ 102), we excluded women ( $n$ 14) who were diagnosed as having GDM already during gestational weeks 8-12. A total of twenty-eight women ( $n$ 28) who had risk factors for GDM or whose fasting venous plasma glucose was $4 \cdot 8-5 \cdot 5 \mathrm{mmol} / \mathrm{l}$ did not wish to participate in the trial for personal or professional reasons ${ }^{(7)}$. We randomised sixty women, of whom three dropped out (10\%) in each group (four early miscarriages, one twin pregnancy and one woman moved away). These high-risk women were randomly assigned to the lifestyle intervention group ( $n$ 27) or to the close follow-up group ( $n$ 27) by the study physician in the Central Hospital with the use of a computed randomisation list. The healthcare nurses who scheduled the study visits did not have access to the randomisation list.

\section{Diet counselling in the lifestyle intervention group}

A clinical nutritionist gave dietary advice tailored to each subject in the lifestyle intervention group individually four times in the first and second trimesters and two times in the third trimester, as described elsewhere ${ }^{(7)}$. Briefly, the dietary goals in this study were: carbohydrate 50-55 energy percent (E\%), fat $30 \mathrm{E} \%$, saturated fat $<10 \mathrm{E} \%$ and protein $15-20 \mathrm{E} \%{ }^{(1,8,9)}$. The aim regarding the intake of dietary fibre was at least $15 \mathrm{~g} / 4184 \mathrm{~kJ}(1000 \mathrm{kcal})$. Women were encouraged to eat a diet rich in vegetables, berries and fruits, and to use fat-free and low-fat dairy products, low-fat meat, soft margarines and vegetable oils (primarily low-erucic acid rapeseed oil) and whole-grain products. Recommendation for energy intake was $126 \mathrm{~kJ} / \mathrm{kg}$ per d for normal-weight women and $105 \mathrm{~kJ} / \mathrm{kg}$ per $\mathrm{d}$ for overweight women. The goal of a during-pregnancy weight gain was $12.5-18 \mathrm{~kg}$ for underweight women, $11.5-16.0 \mathrm{~kg}$ for normal-weight women and $7-11.5 \mathrm{~kg}$ for overweight women ${ }^{(8)}$. The women received information about the goals of eating, demands of pregnancy, meals and snacks, amount of food, supply of fibre, and amount and quality of fats by the nutritionist. The ThreeFactor Eating Questionnaire was used in the beginning of pregnancy and at weeks $36-40^{(10)}$. The baseline $4 \mathrm{~d}$ (four consecutive days, one weekend day) food record was completed before the first appointment at weeks $8-12$, checked by the nutritionist, and formed the basis for dietary advice during the first session. The food record procedure was repeated before the fifth appointment at weeks 26-28 and the sixth appointment at weeks 36-40.

\section{Diet counselling in the close follow-up group}

The women were given general information by a nurse on diet and physical activity in a single session to decrease the risk of GDM during pregnancy. The advice was provided both verbally and in writing. The women returned the Three-Factor Eating Questionnaire in the beginning of pregnancy and at weeks 36-40. The $4 \mathrm{~d}$ food record was completed in the beginning of pregnancy and at weeks 26-28 and 36-40. Otherwise, the women were followed up in the prenatal clinic of the municipal health centre at 1-month intervals according to standard care in Finland ${ }^{(7)}$.

\section{Dietary assessment}

Dietary assessment was carried out by using the Nutrica ${ }^{\circledR}$ nutrient calculation software (Version 2.5; Social Insurance Institute, Turku, Finland) based on Finnish nutrient composition analyses and international food composition tables ${ }^{(11)}$.

The women returned twenty-six food records in the beginning, twenty-two at weeks 26-28 and seventeen in the end of pregnancy in the lifestyle intervention group and twenty-five food records in the beginning and at weeks 26-28 and eighteen in the end of pregnancy in the close follow-up group. The analyses were restricted to the seventeen women in the intervention group and eighteen women in the close followup group who returned all three food records.

\section{Fetal macrosomia}

Birth weight over $4500 \mathrm{~g}$ was defined as fetal macrosomia.

\section{Laboratory assessments}

A $75 \mathrm{~g}, 2 \mathrm{~h}$ oral glucose tolerance test was performed at weeks $8-12$ as previously described elsewhere ${ }^{(7)}$. Blood count was determined with a Sysmex XE-2100 blood count analyser (Sysmex Corporation, Kobe, Japan). Fasting erythrocyte folate, serum- $\mathrm{B}_{12}$-vitamin, serum-thyroxine and serum-thyroid stimulating hormone were all determined using a chemiluminescent microparticle immunoassay method (Abbott Laboratories, 
Abbot Park, IL, USA) in Central Hospital of Seinäjoki. Serum concentrations of total cholesterol, HDL-cholesterol, LDL-cholesterol and TAG were determined using an enzymatic photometric assay (Roche Diagnostics, Basel, Switzerland) in Central Hospital of Seinäjoki. Serum sex hormone-binding globulin was determined with an immunofluorometric assay in the Laboratory MEDIX. Leptin was determined using the Linco RIA method (Linco Research, St Charles, MO, USA) in the Clinical Research Center of University of Eastern Finland.

\section{Statistical analysis}

The final analyses were conducted using SPSS for Windows version 15.0 (SPSS, Inc., Chicago, IL, USA). Macronutrients, micronutrients, the Three-Factor Eating Questionnaire and the weight of women were analysed using repeated-measures ANOVA. Further, the PUFA were analysed using the Student's $t$ test, and corrected using the Bonferroni method. We used the Student's $t$ test for continuous variables and the $\chi^{2}$ or Fisher's exact test for categorical variables. The use of spread was analysed by the McNemar test. Differences were considered significant at a two-sided $P<0.05$.

The power calculation was based on a post hoc analysis of the present results. In the study period, the prevalence of GDM was $23.5 \%$ among the women at high risk of GDM without any interventions in two neighbouring municipalities, whereas the prevalence of GDM in women with early intervention in this study was $7 \cdot 4 \%$. Ultimately, we would have needed a total of 150 (seventy-five per group) highrisk women at a significance level of $5 \%$ and with a power of $80 \%$ to detect a $16 \cdot 1 \%$ difference in the incidence of GDM.

\section{Results}

\section{Characteristics of subjects}

The detailed personal characteristics and baseline macro- and micronutrient intake of the fifty-four women are presented in Table 1. At weeks 26-28, three women were classified as having GDM in the lifestyle intervention group and one in the close follow-up group. None of the women had insulin therapy. Alcohol intake was occasional in both groups during pregnancy. One woman in the intervention group and two women in the close follow-up group smoked during pregnancy.

\section{Eating questionnaire}

There were no differences between the groups in the beginning and end of pregnancy. There was also no effect of intervention except for a slight tendency to additional cognitive restraint in the lifestyle intervention group (Table 2 ).

\section{Dietary intake}

The following macro- and micronutrient intakes were measured: total energy, carbohydrate, fibre, total fat, saturated

Table 1. Baseline characteristics at the beginning of pregnancy

(Mean values and standard deviations)

\begin{tabular}{|c|c|c|c|c|c|}
\hline & \multicolumn{2}{|c|}{$\begin{array}{l}\text { Lifestyle intervention } \\
\quad \text { group }(n 27) \dagger\end{array}$} & \multicolumn{2}{|c|}{$\begin{array}{l}\text { Close follow-up group } \\
\qquad(n \text { 27) } \dagger\end{array}$} & \multirow[b]{2}{*}{$P \neq$} \\
\hline & Mean & SD & Mean & SD & \\
\hline Weight (kg) & $77 \cdot 2$ & $16 \cdot 1$ & $70 \cdot 1$ & 9.5 & 0.073 \\
\hline $\mathrm{BMI}\left(\mathrm{kg} / \mathrm{m}^{2}\right)$ & $27 \cdot 3$ & $6 \cdot 0$ & $25 \cdot 5$ & 3.4 & NS \\
\hline $\mathrm{BMI}>25 \mathrm{~kg} / \mathrm{m}^{2}$ & & & & & NS \\
\hline$n$ & \multicolumn{2}{|c|}{18} & \multicolumn{2}{|c|}{17} & \\
\hline$\%$ & \multicolumn{2}{|c|}{67} & \multicolumn{2}{|c|}{63} & \\
\hline Higher educational status§ & & & & & 0.080 \\
\hline$n$ & \multirow{2}{*}{\multicolumn{2}{|c|}{$\begin{array}{c}5 \\
18.5\end{array}$}} & \multirow{2}{*}{\multicolumn{2}{|c|}{$\begin{array}{c}12 \\
44 \cdot 4\end{array}$}} & \\
\hline$\%$ & & & & & \\
\hline Energy (kJ) & 6346 & 1271 & 6146 & 2210 & NS \\
\hline Carbohydrate (E\%) & $52 \cdot 8$ & 4.5 & 53.3 & $6 \cdot 0$ & NS \\
\hline Fibre $(\mathrm{g})$ & $18 \cdot 2$ & $4 \cdot 8$ & 18.5 & $7 \cdot 1$ & NS \\
\hline Fat $(\mathrm{E} \%)$ & 28.6 & 4.7 & $27 \cdot 0$ & $5 \cdot 7$ & NS \\
\hline Saturated fat (E\%) & 11.3 & $2 \cdot 7$ & $10 \cdot 9$ & $2 \cdot 9$ & NS \\
\hline MUFA (E\%) & 8.9 & 1.8 & 8.6 & $2 \cdot 2$ & NS \\
\hline PUFA (E\%) & 4.5 & $1 \cdot 2$ & 4.5 & 1.6 & NS \\
\hline $18: 2 n-6(E \%)$ & 3.5 & 0.9 & 3.0 & $1 \cdot 1$ & NS \\
\hline $18: 3 n-3(\mathrm{E} \%)$ & 0.7 & 0.2 & 0.6 & 0.2 & $0.040^{*}$ \\
\hline Cholesterol (mg) & 183.6 & 48.9 & $192 \cdot 1$ & $131 \cdot 0$ & NS \\
\hline Protein (E\%) & 18.6 & $2 \cdot 1$ & $19 \cdot 1$ & $2 \cdot 8$ & NS \\
\hline Ascorbic acid (mg) & $127 \cdot 0$ & $56 \cdot 3$ & $147 \cdot 7$ & $100 \cdot 7$ & NS \\
\hline Total vitamin D $(\mu \mathrm{g})$ & 4.1 & $2 \cdot 0$ & 3.9 & $2 \cdot 1$ & NS \\
\hline Folate $(\mu \mathrm{g})$ & $256 \cdot 3$ & 63.3 & $254 \cdot 3$ & $103 \cdot 5$ & NS \\
\hline $\mathrm{Ca}(\mathrm{mg})$ & 1133.0 & $349 \cdot 0$ & $1094 \cdot 0$ & 489.0 & NS \\
\hline $\mathrm{Fe}(\mathrm{mg})$ & 8.7 & 1.8 & 9.0 & 2.9 & NS \\
\hline
\end{tabular}

$\mathrm{E} \%$, percentage of energy.

${ }^{\star} P<0.05$

$\dagger$ Twenty-six of the lifestyle intervention group and twenty-five of the close follow-up group returned the first food record.

$\ddagger$ Student's $t$ test for continuous and $\chi^{2}$ or Fisher's exact test for categorical variables.

$\S$ College or university degree. 
Table 2. The Three-Factor Eating Questionnaire (TFEQ) ${ }^{(10)}$ during pregnancy

(Mean values and standard deviations)

\begin{tabular}{|c|c|c|c|c|c|c|c|c|c|c|}
\hline \multirow{3}{*}{ Gestational weeks... } & \multicolumn{4}{|c|}{ Lifestyle intervention group ( $n$ 27) } & \multicolumn{4}{|c|}{ Close follow-up group ( $n$ 27) } & \multirow[b]{3}{*}{$P^{*}$} & \multirow[b]{3}{*}{$P \dagger$} \\
\hline & \multicolumn{2}{|c|}{$7-12$} & \multicolumn{2}{|c|}{$36-40$} & \multicolumn{2}{|c|}{$7-12$} & \multicolumn{2}{|c|}{$36-40$} & & \\
\hline & Mean & SD & Mean & SD & Mean & SD & Mean & $\mathrm{SD}$ & & \\
\hline $\begin{array}{l}\text { TFEQ§, total score } \\
\text { Min - max. }\end{array}$ & $19 \cdot 8$ & $5-31$ & $19 \cdot 8$ & $8 \cdot 0$ & $19 \cdot 4$ & $6 \cdot 0$ & ${ }_{8}^{18.4}$ & $6 \cdot 1$ & 0.547 & 0.547 \\
\hline $\begin{array}{l}\text { F1: cognitive restraintł } \\
\text { Min. - max. }\end{array}$ & 9.8 & $2-19$ & $10 \cdot 8$ & $5 \cdot 3$ & 11.4 & 4.9 & $10 \cdot 7$ & $5 \cdot 1$ & 0.096 & $0 \cdot 819$ \\
\hline $\begin{array}{l}\text { F2: disinhibition§ } \\
\text { Min.-max. }\end{array}$ & $6 \cdot 1$ & 3.6 & $5 \cdot 2$ & $3 \cdot 3$ & $5 \cdot 1$ & $2 \cdot 6$ & ${ }^{4.9}$ & $3 \cdot 1$ & 0.296 & $0 \cdot 131$ \\
\hline $\begin{array}{l}\text { F3: hunger\| } \\
\text { Min.-max. }\end{array}$ & $3 \cdot 8$ & $2 \cdot 0$ & 3.8 & $2 \cdot 9$ & $0-9$ & $2 \cdot 0$ & $1-9$ & $2 \cdot 3$ & 0.730 & 0.831 \\
\hline
\end{tabular}

Min., minumum; max., maximum; F1, factor 1; F2, factor 2; F3, factor 3.

${ }^{\star} P$ value for the change between groups, fifty-four women returned TFEQ in the beginning and nineteen ( $\left.70 \%\right)$ of the lifestyle intervention group and eighteen (67\%) of close follow-up group in the end of pregnancy.

$\dagger P$ value for the change between weeks $7-12$ and $36-40$, both groups combined.

$\ddagger$ Max. score twenty-one out of fifty-one.

$\S$ Max. score sixteen out of fifty-one.

|| Max. score fourteen out of fifty-one.

fat, MUFA, PUFA, $18: 2 n-6$ (linoleic acid), $18: 3 n$-3 ( $\alpha$-linolenic acid), cholesterol, protein, vitamin $\mathrm{C}$, total vitamin $\mathrm{D}$, folate, $\mathrm{Ca}$ and Fe (Table 3).

The total dietary fat intake met the recommendations, $\leq 30 \mathrm{E} \%$ in both groups. However, the intake of SFA exceeded the recommendations $(<10 \mathrm{E} \%)$ (Table 3$)$. The intake of PUFA increased significantly in the intervention group during pregnancy (repeated-measures ANOVA, $P=0.008$ ) and on average met the recommendations, 5.6 (SD 1.8) E\% (Fig. 1(a)). PUFA intake was statistically significant and greater ( $t$ test, corrected using the Bonferroni method, $P=0.045$ ) at the end of pregnancy. The 18:2n-6 (linoleic acid) intake was systematically higher in the lifestyle intervention group $(P=0.015)$, but there was no statistically significant intervention effect between the groups (Fig. 1(b))

At baseline, $48 \%$ of the women used vegetable oil-based spread, $37 \%$ dairy fat-based spread and 15\% no spread in the lifestyle intervention group. The respective proportions in the close follow-up group were 54, 29 and 17\%. In the end of pregnancy, $90 \%$ of the women in the lifestyle intervention group used vegetable oil-based spread, 5\% dairy fatbased spread and $5 \%$ no spread. The respective proportions in the close follow-up group were 32, 42 and $26 \%$. The use of vegetable oil-based spread increased significantly during pregnancy in the lifestyle intervention group ( $P=0.003$, McNemar test). There was no statistically significant difference in the quality of the spread on the bread during pregnancy in the close follow-up group ( $P=0 \cdot 453$, McNemar test)

The protein intake achieved the recommendations, 15$20 \mathrm{E} \%$ in both groups. The total carbohydrate intake met the recommendations (Table 3). However, the fibre intake remained below the recommendations $>25-35 \mathrm{~g} / \mathrm{d}$ throughout the study, but was at its highest in the second trimester in the lifestyle intervention group, $22 \cdot 1$ (SD 7·1) g/d (Table 3). The total energy intake increased in both groups from the first trimester to the second trimester $(P=0 \cdot 019)$, but was no longer increased in the third trimester (Table 3 ).
The reported dietary intake of folate and Fe and especially vitamin $\mathrm{D}$, only 4.8 (SD 1.8) $\mu \mathrm{g}$, remained below the recommendations in both groups, but dietary $\mathrm{Ca}$ intake met the recommendations (Table 3). In the intervention group, 59\% had some vitamin supplementation in the beginning of pregnancy; most often multivitamin, containing folic acid $100 \mu \mathrm{g}$ and vitamin $\mathrm{D}$ (most commonly $\mathrm{D}_{3}$ ) $5 \mu \mathrm{g}, 1-2$ tablets/d. In the close follow-up group, 33\% took vitamin supplements. Vitamin supplementation was not reported systematically during pregnancy.

\section{Weight gain}

The intensive dietary education resulted in a somewhat lower weight gain ( $P=0.062$, adjusted by the pre-pregnancy weight) as compared to the close follow-up group at the end of pregnancy (Table 4).

\section{Mean birth weight}

The mean birth weight was greater, 3871 (SD 567) g, in the lifestyle intervention group $(P=0 \cdot 047$, adjusted by the prepregnancy weight of the women) compared with the close follow-up group, 3491 (SD 573) g. The ranges of birth weights were $2870-5275 \mathrm{~g}$ in the intervention group and $2390-5050 \mathrm{~g}$ in the close follow-up group. There was no difference in the gestational age: $39 \cdot 8$ (SD 1.2) weeks in the intervention group and 39.6 (SD 1.9) weeks in the close follow-up group. The mean birth weight was $3564 \mathrm{~g}$ in the period 1996-2000 in Finland ${ }^{(12)}$. There was no difference in macrosomia ( $P=0 \cdot 480$, adjusted by the pre-pregnancy weight of the women) between the groups.

\section{Laboratory assessments}

There were no differences between the assessments of blood count, fasting erythrocyte folate, serum-B $\mathrm{B}_{12}$-vitamin, 
Table 3. Intake of macronutrients and micronutrients in the lifestyle intervention and close follow-up groups during pregnancy (Mean values and standard deviations)

\begin{tabular}{|c|c|c|c|c|c|c|c|c|c|c|c|c|c|c|}
\hline \multirow{3}{*}{ Gestational weeks... } & \multicolumn{6}{|c|}{ Lifestyle intervention group ( $n$ 27) } & \multicolumn{6}{|c|}{ Close follow-up group ( $n$ 27) } & \multirow[b]{3}{*}{ Recommendation $†$} & \multirow[b]{3}{*}{$P \ddagger$} \\
\hline & \multicolumn{2}{|c|}{$7-12(n 26)$} & \multicolumn{2}{|c|}{$26-28$ ( $n 22)$} & \multicolumn{2}{|c|}{$36-40(n 17)$} & \multicolumn{2}{|c|}{$7-12(n 25)$} & \multicolumn{2}{|c|}{$26-28(n 25)$} & \multicolumn{2}{|c|}{$36-40(n 18)$} & & \\
\hline & Mean & SD & Mean & SD & Mean & SD & Mean & SD & Mean & SD & Mean & SD & & \\
\hline Energy (kJ) & 6346 & 1271 & 6725 & 1616 & 6707 & 1291 & 6146 & 2210 & 7282 & 2465 & 7296 & 2900 & $\S$ & NS \\
\hline Carbohydrate (E\%) & $52 \cdot 8$ & 4.5 & $52 \cdot 6$ & $5 \cdot 8$ & $53 \cdot 2$ & 4.4 & $53 \cdot 3$ & $6 \cdot 0$ & $52 \cdot 4$ & $7 \cdot 0$ & $55 \cdot 6$ & $6 \cdot 7$ & $50-60$ & NS \\
\hline Fibre $(g)$ & $18 \cdot 2$ & 4.8 & $22 \cdot 3$ & $7 \cdot 4$ & $19 \cdot 6$ & 6.4 & 18.5 & $7 \cdot 1$ & $20 \cdot 8$ & 7.5 & $19 \cdot 3$ & $7 \cdot 7$ & $25-35$ & NS \\
\hline Total fat (E\%) & 28.6 & 4.7 & $29 \cdot 4$ & $6 \cdot 2$ & $29 \cdot 1$ & 4.7 & $27 \cdot 0$ & $5 \cdot 7$ & $29 \cdot 3$ & 6.4 & $26 \cdot 1$ & $6 \cdot 0$ & 30 & NS \\
\hline Saturated fat (E\%) & $11 \cdot 3$ & $2 \cdot 7$ & $10 \cdot 5$ & $2 \cdot 3$ & $11 \cdot 3$ & $2 \cdot 9$ & $10 \cdot 9$ & $2 \cdot 9$ & $12 \cdot 1$ & $3 \cdot 6$ & $11 \cdot 0$ & $2 \cdot 9$ & 10 & NS \\
\hline Unsaturated fat (E\%) & 13.4 & $2 \cdot 7$ & $15 \cdot 3$ & 4.5 & $14 \cdot 3$ & $3 \cdot 1$ & $13 \cdot 1$ & 3.4 & $13 \cdot 6$ & 3.4 & 11.8 & $3 \cdot 7$ & $20-25$ & NS \\
\hline MUFA (E\%) & 8.9 & 1.8 & $9 \cdot 4$ & $2 \cdot 3$ & 8.7 & 1.6 & $8 \cdot 6$ & $2 \cdot 2$ & $9 \cdot 1$ & $2 \cdot 2$ & 7.9 & $2 \cdot 3$ & $10-15$ & NS \\
\hline PUFA (E\%) & 4.5 & $1 \cdot 2$ & 5.9 & $2 \cdot 4$ & $5 \cdot 6$ & 1.8 & 4.5 & $1 \cdot 6$ & 4.5 & 1.5 & 3.9 & 1.8 & $5-10$ & $0.008^{* *}$ \\
\hline $18: 2 n-6(E \%)$ & 3.5 & 0.9 & 4.2 & 1.5 & $4 \cdot 1$ & 1.3 & 3.0 & $1 \cdot 1$ & $3 \cdot 3$ & $1 \cdot 2$ & $2 \cdot 8$ & 1.4 & 3 & NS \\
\hline $18: 3 n-3(\mathrm{E} \%)$ & 0.7 & 0.2 & 0.8 & 0.3 & 0.6 & 0.2 & 0.6 & 0.2 & 0.6 & 0.2 & 0.5 & 0.3 & 1 & NS \\
\hline Cholesterol (mg) & 183.6 & 48.9 & 164.9 & 49.8 & $185 \cdot 2$ & 49.5 & $192 \cdot 1$ & $131 \cdot 0$ & 193.9 & 85.9 & $195 \cdot 1$ & $97 \cdot 7$ & & NS \\
\hline Protein (E\%) & $18 \cdot 6$ & $2 \cdot 1$ & $18 \cdot 0$ & $2 \cdot 3$ & $17 \cdot 7$ & $2 \cdot 4$ & $19 \cdot 1$ & $2 \cdot 8$ & $18 \cdot 1$ & $3 \cdot 2$ & $18 \cdot 3$ & 3.3 & 15 & NS \\
\hline Ascorbic acid (mg) & $127 \cdot 0$ & $56 \cdot 3$ & $132 \cdot 2$ & $96 \cdot 7$ & $105 \cdot 0$ & 39.0 & $147 \cdot 7$ & $100 \cdot 7$ & $156 \cdot 1$ & $82 \cdot 2$ & $175 \cdot 0$ & $128 \cdot 9$ & 85 & NS \\
\hline Total vitamin D $(\mu \mathrm{g})$ & $4 \cdot 1$ & $2 \cdot 0$ & 4.7 & 1.6 & 4.8 & 1.8 & 3.9 & $2 \cdot 1$ & 3.8 & 1.7 & 4.4 & $2 \cdot 1$ & 10 & NS \\
\hline Folate $(\mu \mathrm{g})$ & $256 \cdot 3$ & 63.3 & $264 \cdot 1$ & $72 \cdot 6$ & $253 \cdot 1$ & 44.7 & $254 \cdot 3$ & 103.5 & $291 \cdot 3$ & $94 \cdot 3$ & 294.7 & $105 \cdot 3$ & 400 & NS \\
\hline $\mathrm{Ca}(\mathrm{mg})$ & 1133 & 349 & 1104 & 297 & 1191 & 389 & 1094 & 489 & 1234 & 430 & 1289 & 464 & 900 & NS \\
\hline $\mathrm{Fe}(\mathrm{mg})$ & 8.7 & 1.8 & 9.5 & $2 \cdot 8$ & 9.4 & $3 \cdot 2$ & $9 \cdot 0$ & 2.9 & $10 \cdot 7$ & 4.8 & $9 \cdot 8$ & 3.0 & 15 & NS \\
\hline
\end{tabular}

E\%, percentage of energy.

${ }^{* *} P<0.01$.

†DNSG, the Diabetes and Nutrition Study Group of EASD ${ }^{(9)}$, DPS, Diabetes Prevention Study ${ }^{(1)}$, Ministry of Social Affairs and Health $2004^{(8)}$

$\ddagger P$ value effect of intervention between groups (repeated-measures ANOVA), seventeen of intensive lifestyle group and eighteen of close follow-up group returned all three food records.

$\S$ Recommendation of total energy was $126 \mathrm{~kJ} / \mathrm{kg}$ per $\mathrm{d}$ for women with normal weight and $105 \mathrm{~kJ} / \mathrm{kg}$ per $\mathrm{d}$ for women with overweight. 
(a)

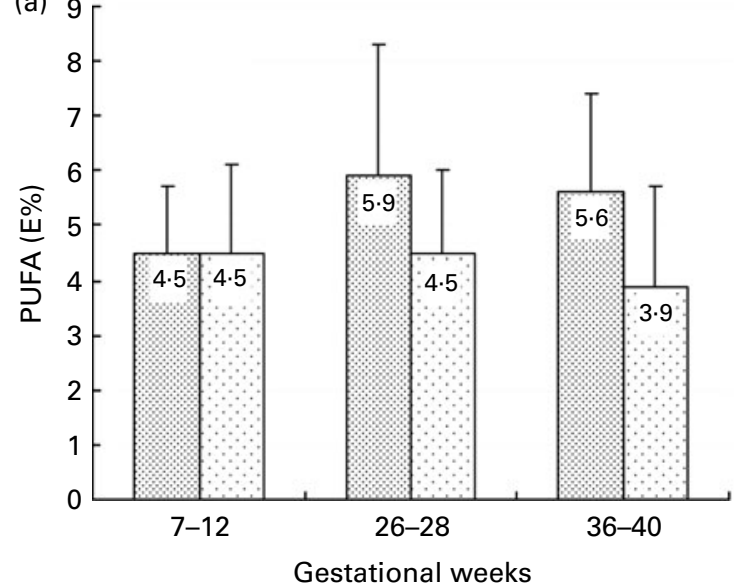

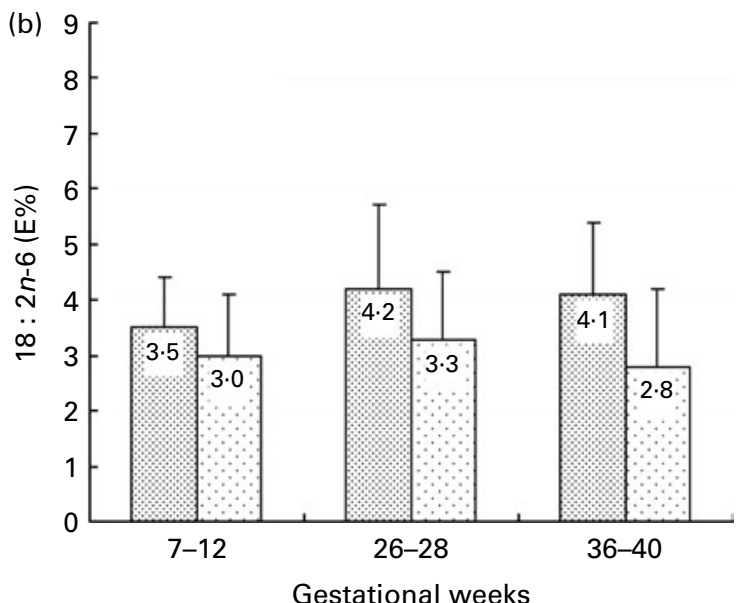

Gestational weeks

Fig. 1. Dietary intake of (a) PUFA (intervention effect, $P=0.008$ ) and (b) $18: 2 n-6$ (systematic difference, $P=0.015)$ during pregnancy in the intervention and follow-up groups (repeated-measures ANOVA). Values are means and standard deviations represented by vertical bars. E\%, percentage of energy. $E$, Lifestyle intervention; $匚$, close follow-up.

serum-free-thyroxine, serum-thyrotrophin, serum sex hormone-binding globulin, total cholesterol, HDL-cholesterol, LDL-cholesterol, TAG, umbilical cord-leptin or in glucose tolerance between the groups. The only statistically significant difference was the higher number of erythrocytes $(P=0.035)$ in the lifestyle intervention group (data not shown). Nobody had anaemia, $\mathrm{Hb} \leq 100 \mathrm{~g} / \mathrm{l}$. Two women in the intervention group and three in the control group had $\mathrm{Hb} \leq 105 \mathrm{~g} / \mathrm{l}$. There was no difference observed in serum lipids, serum thyroid stimulating hormone or serum-free-thyroxin between the groups. In both groups, serum concentrations of total and LDL-cholesterol, HDL-cholesterol and TAG were higher $(P<0.001)$ at the end of pregnancy than in the first trimester, respectively. Serum-free-thyroxin was lower $(P<0.001)$ and serum thyroid stimulating hormone higher $(P=0.002)$ in the third trimester in both groups (data not shown). There was no difference in cord-leptin between the groups.

\section{Discussion}

A lifestyle intervention since early pregnancy with an individualised dietary counselling by a clinical nutritionist improved the quality of dietary fat during pregnancy. Weight gain of the women in the intervention group was somewhat lower (although not statistically significant) and birth weight of the infants was higher, although within normal range. The occurrence of macrosomia was similar between the groups. Dietary studies are complex during pregnancy, because the macroand micronutrient requirements keep changing constantly. This may explain the reason for the lack of controlled dietary intervention studies.

In the present study, energy intake of the women at a high risk of GDM increased $6 \%$ in the lifestyle intervention group and $18 \%$ in the close follow-up group during the second trimester, but no further during the third trimester. This is somewhat lesser than what may be expected, given the $11-14 \mathrm{~kg}$ weight gain that occurred during pregnancy. Extra energy intake is required by pregnant women to support adequate gestational weight gain and increase in BMR, which are not totally offset by reductions in the physical exercise-related energy expenditure. In a previous study, objectively measured energy intake increased 7, 16 and $38 \%$ in the first, second and third trimesters, respectively, in the high BMI group compared with normal-weight women ${ }^{(13)}$. Underreporting of energy consumption by self-report is well recognised, linked to increased adiposity and body size, dietary restraint and

Table 4. Energy intake and weight of women during pregnancy

(Mean values and standard deviations)

\begin{tabular}{|c|c|c|c|c|c|c|c|c|c|c|c|c|c|}
\hline \multirow{3}{*}{ Gestational weeks... } & \multicolumn{6}{|c|}{ Lifestyle intervention group ( $n$ 27) } & \multicolumn{6}{|c|}{ Close follow-up group ( $n$ 27) } & \multirow[b]{3}{*}{$P^{*}$} \\
\hline & \multicolumn{2}{|c|}{$7-12$} & \multicolumn{2}{|c|}{$26-28$} & \multicolumn{2}{|c|}{$36-40$} & \multicolumn{2}{|c|}{$7-12$} & \multicolumn{2}{|c|}{$26-28$} & \multicolumn{2}{|c|}{$36-40$} & \\
\hline & Mean & SD & Mean & SD & Mean & SD & Mean & SD & Mean & SD & Mean & SD & \\
\hline Weight (kg) & \multirow[t]{3}{*}{$77 \cdot 2$} & \multirow[t]{3}{*}{$16 \cdot 1$} & 83.2 & $14 \cdot 6$ & 88.6 & $14 \cdot 4$ & \multirow[t]{3}{*}{$70 \cdot 1$} & \multirow[t]{3}{*}{$9 \cdot 5$} & 78.5 & $10 \cdot 2$ & $84 \cdot 1$ & $11 \cdot 3$ & \multirow{3}{*}{$\begin{array}{c}0.062 \\
\text { NS }\end{array}$} \\
\hline Weight gain $(\mathrm{kg})$ & & & 6.0 & 3.6 & 11.4 & $6 \cdot 0$ & & & 8.4 & 3.6 & 13.9 & $5 \cdot 1$ & \\
\hline Min. - max. $(\mathrm{kg})$ & & & & & $2 \cdot 2$ & $28 \cdot 8$ & & & & & $4 \cdot 6$ & $26 \cdot 8$ & \\
\hline Energy $(\mathrm{kJ}) \dagger$ & 6346 & 1271 & 6725 & 1616 & 6707 & 1291 & 6146 & 2210 & 7282 & 2465 & 7296 & 2900 & NS \\
\hline Min. - max. & \multicolumn{2}{|c|}{$4412-8685$} & \multicolumn{2}{|c|}{$3755-9410$} & \multicolumn{2}{|c|}{$4547-8959$} & \multicolumn{2}{|c|}{$3053-12483$} & \multicolumn{2}{|c|}{$4201-13082$} & \multicolumn{2}{|c|}{$4514-16075$} & \\
\hline
\end{tabular}

Min., minimum; max., maximum

${ }^{*} P$ value for the change between groups (repeated-measures ANOVA adjusted by the pre-pregnancy weight).

$\dagger$ In the lifestyle intervention group seventeen and in the close follow-up group eighteen women were analysed. 
socio-economic status ${ }^{(14,15)}$, and women with a high prepregnancy BMI are more likely to underreport nutrient intakes $^{(16,17)}$. Overall, $65 \%$ of women of the present study were overweight $\left(\mathrm{BMI}>25 \mathrm{~kg} / \mathrm{m}^{2}\right.$ ) before pregnancy. The weight gain tended to be lower in the lifestyle intervention group. Obese women, on average, gain less weight during pregnancy than women with normal weight ${ }^{(18)}$. The women in the intervention group were less educated and overweight was more common. Only one of the women out of four weighing over $100 \mathrm{~kg}$ returned all three diaries. It seems probable that those women who failed to meet the aims of the intervention were most likely to refuse the reporting.

The mean birth weight in the lifestyle intervention group was greater than in the close follow-up group $(P=0.047)$, but macrosomia did not differ between the groups. Avoidance of macrosomia is an important goal in treating women with GDM. On the other hand, Lammi et al. ${ }^{(19)}$ showed in a population-based case-control study that the risk of early-onset type 2 diabetes decreased with increasing birth weight until $4.2 \mathrm{~kg}$. Because the mean birth weight in the intervention group was well under $4 \mathrm{~kg}$, the increase in birth weight may be of benefit for most offspring with respect to risk of type 2 diabetes and other chronic diseases associated with low birth weight. Consistent evidence for an influence of women's dietary composition during pregnancy on fetal growth is lacking. Moore et al. ${ }^{(20)}$ have studied in a prospective, observational research that the $\mathrm{E} \%$ from carbohydrate in early and late pregnancy was negatively associated, and protein intake from dairy sources was positively associated with ponderal index of the baby. Godfrey et al. ${ }^{(21)}$ showed an association between the carbohydrate intake of mothers in early pregnancy with methylation of genes, predicting adiposity in children at 9 years of age. This study indicated that a mother's dietary habits during pregnancy have long-lasting effects on the child, possibly by epigenetic mechanisms ${ }^{(21)}$.

Overall, the macronutrient intake in both groups met the goals of the study: carbohydrate $50-55 \mathrm{E} \%$, fat $\leq 30 \mathrm{E} \%$ and protein $15-20 \mathrm{E} \%$. We noted relatively small changes in the dietary intake, except for the increase in PUFA and 18:2n-6 (linoleic acid) in the lifestyle intervention group counselled by the clinical nutritionist. The vegetable oil-based spread substituted for a dairy fat-based spread in the lifestyle intervention group was the most evident explanatory factor. In the third trimester, the women in the close follow-up group reduced the intake of total fat, including unsaturated fat. Women in the intervention group maintained the intake of unsaturated fat throughout pregnancy such that the intake of unsaturated fat was statistically significant and greater $(P=0.034)$ than in the close follow-up group in the end of pregnancy. In the Finnish diet, the use of visible fat as a source of unsaturated fatty acids (spread, salad dressing and cooking oils) is essential in ensuring the recommended fatty acid composition of a diet. In the present study, the subjects were able to change the spread used on the bread, but other recommended changes in dietary fat intake were not adopted. Saturated fat intake remained above the dietary goal of $<10 \mathrm{E} \%$ in both groups, despite a transient decrease in the lifestyle intervention group and increase in the close follow-up group during the second trimester. Fibre intake remained below the recommendation of $>25-35 \mathrm{~g} / \mathrm{d}$, at its best $22 \mathrm{~g} / \mathrm{d}$ in the lifestyle intervention group in mid-pregnancy.

The women in the lifestyle intervention group adopted healthier eating habits. Many dietary outcomes such as intake of fibre, saturated fat, unsaturated fat, MUFA, PUFA, $18: 2 n-6$ and cholesterol were closest to optimal in midpregnancy (Table 3). However, the women were not able to maintain changes in eating habits to the end of pregnancy, except for vegetable oil-based fat products. In the close follow-up group, total fat intake decreased (including PUFA) during the study. This result shows how difficult it is to optimise the quality of fat in the diet without professional counselling in a country where the quality of fat is traditionally more saturated than recommended. In this small sample ( $n$ 17), we could not find any factor other than education that was related to a favourable intervention response. It could be worthwhile to explore the effects of other approaches, such as educational group sessions, to achieve more persistent changes in dietary patterns.

The Three-Factor Eating Questionnaire ${ }^{(10)}$ was used in this feasibility study for measuring three dimensions (cognitive restraint of eating, disinhibition and hunger) of eating behaviour. The questionnaire assists in selecting the most appropriate method of counselling for the patient. The benefit of this questionnaire was limited in the present study, because all women received informative education regardless of the results of the questionnaire. Women with a high cognitive restraint score benefit from informative education, but individuals with a high disinhibition score may be more suitable for behavioural management or peer-group approaches, especially when dealing with emotional disinhibitors such as anxiety, depression or loneliness ${ }^{(10)}$. In this study, three mothers had high scores on hunger (8-9 out of 14) in the beginning of pregnancy and five mothers (8-10 out of 14) in the end of pregnancy. Hunger is by far the most important factor regulating the eating of women in Finland, based on the present study.

Even taking into account underreporting, dietary vitamin D intake, only 4.8 (SD 1.8$) \mu \mathrm{g}$, is clearly below the recommendations $(10 \mu \mathrm{g})$. Fish meals were random in food records and the vitamin supplementation was not reported systematically during pregnancy. Serum vitamin D concentrations were not assessed. The intakes of vitamin $\mathrm{D}$ and folate were below the recommendations in Finland, also in the studies of Arkkola et $a l .{ }^{(22)}$ and Järvenpää et $a l .{ }^{(23)}$. The reported intakes of folate and Fe were below the recommendations. Fasting erythrocyte folate and the blood count were normal, however, which may suggest underreporting. Food records over $4 \mathrm{~d}$, which provide a reliable estimate of macronutrient intake, may nonetheless be insufficient for the estimation of micronutrient intake ${ }^{(24)}$.

The purpose of the laboratory assessments was to show that the possible changes in the diet are not harmful to women and do not cause deficiencies of macro- or micronutrients. There were no differences between the groups in glucose tolerance or other laboratory assessments. Free-thyroxin initially increased, peaking between 9 and 13 weeks ${ }^{(25)}$ and then decreased; thyrotrophin mirrored changes in free-thyroxin. 
During pregnancy, the concentrations of total cholesterol and HDL- and LDL-cholesterol and TAG increased dramatically in both groups $(P<0 \cdot 001)$. These are part of the natural metabolic changes in pregnancy, which seem to occur irrespective of the qualitative or quantitative changes in dietary fat intake. The implications of these changes are unclear ${ }^{(26)}$, but they may be important for the fetus, especially for the development of brain and other tissues.

\section{Conclusions}

Individualised counselling by a clinical nutritionist as part of a lifestyle intervention improved the quality of dietary fat intake in pregnant women at a high risk of GDM. Birth weight was higher in the intervention group, but there was no difference in macrosomia. It may be beneficial to test other approaches, such as peer-group sessions, to achieve broader, longer-lasting changes in dietary intake.

\section{Acknowledgements}

The present study was funded by Seinäjoki Central Hospital and Kuopio University Hospital, University of Eastern Finland and the municipalities of Kauhajoki and Lapua, i.e. employers of the authors. The study was supported by EVO funding from Kuopio University Hospital and South Ostrobothnia Hospital District. The authors declare that they have no conflicts of interest. E. K.-H. participated in the design of the study, acquisition of the data, performed the statistical analysis and drafted the manuscript. U. S., D. E. L., L. N. and S. H. made substantial contributions to the conception and design of the study, and data interpretation, and helped in the drafting of the manuscript. H. L. participated in data collection and dietary counselling of the subjects. All authors read and approved the final manuscript.

\section{References}

1. Tuomilehto J, Lindström J, Eriksson JG, et al. (2001) Prevention of type 2 diabetes mellitus by changes in lifestyle among subjects with impaired glucose tolerance. N Engl J Med $\mathbf{3 4 4}$, 1343-1350.

2. Diabetes Prevention Program Research Group (2002) Reduction in the incidence of type 2 diabetes with lifestyle intervention or metformin. N Engl J Med 346, 393-403.

3. Moses RG, Shand JL \& Tapsell LC (1997) The recurrence of gestational diabetes: could dietary differences in fat intake be an explanation? Diabetes Care 20, 1635-1637.

4. Wang Y, Storlien LH, Jenkins AB, et al. (2000) Dietary variables and glucose tolerance in pregnancy. Diabetes Care 23, 460-464.

5. Bo S, Menato G, Lezo A, et al. (2001) Dietary fat and gestational hyperglycaemia. Diabetologia 44, 972-978.

6. Saldana TM, Siega-Riz AM \& Adair LS (2004) Effect of macronutrient intake on the development of glucose intolerance during pregnancy. Am J Clin Nutr 79, 479-486.

7. Korpi-Hyövälti E, Laaksonen DE, Schwab U, et al. (2011) Feasibility of a lifestyle intervention in early risk pregnancy to prevent deterioration of glucose tolerance. BMC Public Health 11, 179.
8. Hasunen K, Kalavainen M, Keinonen H, et al. (2004) Lapsi, perhe ja ruoka (Child, Family and Food). Dietary Recommendations for Children and Pregnant and Lactating Women (in Finnish). Publication of Ministry of Social Affairs and Health, vol. 11, pp. 70-89. Helsinki: Edita Prima Oy.

9. The Diabetes and Nutrition Study Group (DNSG) of the European Association for the Study of Diabetes (EASD) (2000) Recommendations for the nutritional management of patients with diabetes mellitus. Eur J Clin Nutr $\mathbf{5 4}$, $353-355$.

10. Stunkard AJ \& Messick S (1985) The Three-Factor Eating Questionnaire to measure dietary restraint, disinhibition and hunger. J Psychosom Res 29, 71-73.

11. Rastas M, Seppänen R, Knuts L-R, et al. (1997) Nutrient Composition of Foods. Turku, Finland: The Social Insurance Institution.

12. Mortensen LH, Diderichsen F, Amtzen A, et al. (2008) Social inequality in fetal growth: a comparative study of Denmark, Finland, Norway and Sweden in the period 1981-2000. J Epidemiol Community Health 62, 325-331.

13. Butte NF, William WW, Treuth MS, et al. (2004) Energy requirements during pregnancy based on total energy expenditure and energy deposition. Am J Clin Nutr 79, 1078-1087.

14. Hill RJ \& Dawies PSW (2001) The validity of self reported energy intake as determined using the doubly labelled water technique. Br J Nutr 85, 415-430.

15. Black AE \& Cole TJ (2001) Biased over- or under-reporting is characteristic of individuals whether over time or by different assessment methods. J Am Diet Assoc 101, 70-80.

16. Olafsdottir AS, Thorsdottir I, Gunnarsdottir I, et al. (2006) Comparison of women's diet assessed by FFQs and 24hour recalls with and without underreporters: associations with biomarkers. Ann Nutr Metab 50, 450-460.

17. Derbyshire E, Davies J, Costarelli V, et al. (2006) Prepregnancy body mass index and dietary intake in the first trimester of pregnancy. J Hum Nutr Diet 19, 267-273.

18. Carmichael S, Abrams B \& Selvin S (1997) The pattern of maternal weight gain in women with good pregnancy outcomes. Am J Public Health 87, 1984-1988.

19. Lammi N, Blomstedt PA, Moltchanova E, et al. (2009) Perinatal risk factors in young adult-onset type 1 and type 2 diabetes - a population-based case-control study. Acta Obstet Gynecol Scand 88, 468-674.

20. Moore VM, Davies MJ, Willson KJ, et al. (2004) Dietary composition of pregnant women is related to size of the baby at birth. J Nutr 134, 1820-1826.

21. Godfrey KM, Sheppard A, Gluckman PD, et al. (2011) Epigenetic gene promoter methylation at birth is associated with child's later adiposity. Diabetes 60, 1528-1534.

22. Arkkola T, Uusitalo U, Pietikäinen M, et al. (2006) Dietary intake and use of dietary supplements in relation to demografic variables among pregnant Finnish women. Br J Nutr 96, 913-920.

23. Järvenpää J, Schwab U, Lappalainen T, et al. (2007) Fortified mineral water improves folate status and decreased plasma homocysteine concentration in pregnant women. J Perinat Med 35, 108-114.

24. Basiotis PP, Welsh SO, Cronin FJ, et al. (1987) Number of days of food intake records required to estimate individual and group nutrient intakes with defined confidence. $J$ Nutr 117, 1638-1641.

25. Panesar NS, Li CY \& Rogers MS (2001) Reference intervals for thyroid hormones in pregnant Chinese women. Ann Clin Biochem 38, 329-332.

26. Martin U, Davies C, Hayavi S, et al. (1999) Is normal pregnancy atherogenic? Clin Sci 96, 421-425. 